\title{
Nota CienTíFICA \\ Efecto de la temperatura y salinidad en el crecimiento larval de Litopenaeus vannamei
}

Effect of temperature and salinity on larval growth of Litopenaeus vannamei

\author{
J. Francisco Bermudes-Lizárraga ${ }^{1}$, Mario Nieves-Soto ${ }^{1,2}$, M. Alejandra Medina-Jasso2, \\ J. Cristobal Román-Reyes ${ }^{1,2}$, Luis M. Flores-Campaña ${ }^{1,2}$, Armando A. \\ Ortega-Salas $^{3}$ y Pablo Piña-Valdez ${ }^{1,2}$
}

\begin{abstract}
${ }^{1}$ Programa de Doctorado en Ciencias en Recursos Acuáticos, Facultad de Ciencias del Mar, Universidad Autónoma de Sinaloa, Av. de los Deportes s/n, C.P. 82017, Mazatlán, Sinaloa, México. pablopina@live.com.mx

${ }^{2}$ Facultad de Ciencias del Mar, Universidad Autónoma de Sinaloa, Paseo Claussen s/n, C.P. 82000, Mazatlán, Sinaloa, México

${ }^{3}$ Instituto de Ciencias del Mar y Limnología, Universidad Nacional Autónoma de México, Calzada Joel Montes Camarena s/n, C.P. 82040, Mazatlán, Sinaloa, México
\end{abstract}

\begin{abstract}
The purpose of this study was to evaluate the effect of 12 combinations of salinity and temperature on larval growth of Litopenaeus vannamei, starting in nauplius $\mathrm{V}$ (NV) and ending at postlarva (PL1), a process which lasted seven days. The results show that the larvae have greater length and weight at temperatures of 30 to $35^{\circ} \mathrm{C}$ in combination with salinities between 25 and 35. In conclusion, salinity and temperature are important factors to consider for the production of larvae of L. vannamei.
\end{abstract}

Key words: Environmental parameters, shrimp, larvae, Litopenaeus vannamei

\section{INTRODUCCIÓN}

En el caso de los camarones que desarrollan parte de su ciclo de vida en ambientes estuarinos, como la población migratoria de Litopenaeus vannamei (Boone, 1931), la salinidad y la temperatura son las variables más influyentes en el metabolismo, desarrollo y crecimiento de larvas (Pham et al. 2012, ChongRobles et al. 2014, Piña-Valdez et al. 2015). Los organismos de esta especie poseen gran capacidad osmorregulatoria entre las diferentes etapas de desarrollo. Las larvas migran del océano a las lagunas costeras quedando expuestas a variaciones de salinidad y temperatura hasta convertirse en adultos (ChongRobles et al. 2014).

Existen pocos estudios sobre el crecimiento larval y tolerancia a cambios amplios de salinidad en los laboratorios productores de larvas del noroeste de México (Chong-Robles et al. 2014, Jamali et al. 2015). El efecto combinado de temperaturasalinidad no ha sido investigado en larvas de L. vannamei; mientras que en especies asiáticas hay pocos trabajos enfocados a determinar condiciones óptimas de estas variables ambientales (Kumlu et al. 2000, Zacharia \& Kakati 2004).

Actualmente, no se tiene información de los factores abióticos más importantes para la cría de esta especie (MagallónBarajas et al. 2006, Chong-Robles et al. 2014). Esta información es importante debido al prominente desarrollo de la camaronicultura epicontinental en aguas de baja salinidad (Godínez-Siordia et al. 2011), así como en aguas de altas salinidades y temperaturas, donde se han presentado dificultades en la producción del camarón (Laramore et al. 2009, HoangOanh et al. 2013). Por tanto, en el presente trabajo se evaluó el efecto combinado de 3 temperaturas $\left(25,30\right.$ y $\left.35^{\circ} \mathrm{C}\right)$ y 4 salinidades $(25,30,35$ y 40$)$ sobre la longitud y el peso de $L$. vannamei desde la etapa larvaria de nauplio $\mathrm{V}$ a poslarva. $\mathrm{La}$ hipótesis de trabajo fue que las larvas de L. vannamei por habitar en ambientes oceánicos y estuarinos poseen la capacidad de crecer en amplias variaciones de salinidad y temperatura.

\section{MateRiales Y MÉTODOS \\ Cultivo de APOyo}

Las larvas de camarón en la fase de nauplio IV, se obtuvieron del laboratorio de producción comercial 'FITMAR' ubicado en el sur de Sinaloa, México. Las larvas fueron aclimatadas y transportadas al laboratorio mediante las técnicas utilizadas por Medina-Jasso et al. (2015).

Las larvas zoea de L. vannamei fueron alimentadas con la microalga Chaetoceros muelleri, la cual fue obtenida del cepario del Laboratorio de Ecofisiología de Organismos Acuáticos y Cultivos de Apoyo de la Facultad de Ciencias del Mar de la Universidad Autónoma de Sinaloa. Las microalgas se cultivaron de acuerdo a la técnica de Piña et al. (2006). Durante la fase de mysis los organismos se alimentaron con nauplios de Artemia y microalgas. Los quistes de Artemia se adquirieron de una empresa comercial y su eclosión se realizó de acuerdo a Rodríguez-Canché et al. (2006). 


\section{DiSEÑO EXPERIMENTAL}

Una vez alcanzada la fase nauplio $\mathrm{V}$ en un $100 \%$, se dio inicio al experimento con 12 tratamientos: combinación de 4 salinidades $(25,30,35$ y 40$)$ y 3 temperaturas $\left(25,30\right.$ y $\left.35^{\circ} \mathrm{C}\right)$, cada tratamiento por cuatriplicado. Se utilizaron recipientes de plástico con $12 \mathrm{~L}$ de agua de mar a salinidad de 35 filtrada a 1 $\mu \mathrm{m}$ y una densidad inicial de 100 larvas $\mathrm{L}^{-1}$. Las temperaturas se mantuvieron con un calentador de titanio (FINNEX, HMO50) por acuario. Para incrementar la salinidad a 40 se añadió sal granulada (sin yodo) a razón de $5 \mathrm{~g} \mathrm{~L}^{-1}$, y para disminuirla se agregó agua dulce filtrada a $1 \mu \mathrm{m}$. La salinidad se midió en los tratamientos con un refractómetro digital (VITALSINE, SR6).

Las larvas fueron alimentadas en una sola ración diaria de acuerdo a las cantidades señaladas por Medina-Jasso et al. (2015). Diariamente se hicieron recambios de agua (50\%) previamente se ajustó la temperatura y la salinidad correspondiente a cada unidad experimental.

Para determinar el efecto de la salinidad y la temperatura sobre el crecimiento se tomó inicialmente una muestra de 15 larvas para determinar la longitud y otra de 1.000 organismos para la biomasa orgánica de la fase de nauplio $\mathrm{V}$, que fueron de $0,53 \pm 0,01 \mathrm{~mm}$ y de $2,18 \pm 0,13 \mu \mathrm{g}$ larva ${ }^{-1}$ respectivamente. Asimismo, al término del experimento se tomaron muestras de 12-15 larvas de cada acuario para evaluar la longitud total (LT), las mediciones se realizaron de acuerdo a Piña et al. (2006) y Jamali et al. (2015). Para obtener el peso orgánico final se concentraron un máximo de 400 organismos de cada acuario en filtros de fibra de vidrio Whatman GF-C de acuerdo a estudios previos (Piña et al. 2006, Medina-Jasso et al. 2015, Piña-Valdez et al. 2015).

\section{Análisis estadístico}

A los datos de longitud y peso, se les aplicaron las pruebas de normalidad (Lilliefors) y de igualdad de varianzas (Bartlett). Posteriormente se analizaron por un ANDEVA de dos factores, considerando a la salinidad el factor A, la temperatura el factor B. Cuando se obtuvieron diferencias significativas entre las medias de los tratamientos, se realizaron pruebas de comparaciones múltiples de Tukey, utilizando un nivel de significancia $(\alpha)$ de 0.05 (Zar 2010).

\section{RESULTADOS Y DISCUSIÓN}

El ANDEVA mostró que ambos factores, la salinidad y la temperatura, influyeron significativamente sobre la longitud total y el peso orgánico final. En el caso de la LT al considerar la salinidad en el ANDEVA se tuvo un F=14,90 para una $P<$ 0,0005 , y con la temperatura el ANDEVA produjo un $\mathrm{F}=$ 107,66 para una $P<0,0005$, sin embargo la interacción de ambos factores no mostró significancia (ANDEVA F = 0,96 para una $P>0,25$ ) (Tabla 1A). De igual manera el ANDEVA aplicado a los datos de los pesos orgánicos demostró que la salinidad, la temperatura y su interacción afectaron significativamente $(P<0,0005)$ la biomasa orgánica promedio final de los diferentes tratamientos ensayados; así en el ANDEVA con la salinidad se evaluó un $\mathrm{F}=44,04$, con la temperatura un $\mathrm{F}=625,41$ y con la interacción un $\mathrm{F}=20,12$ (Tabla 1B).

Tabla 1. ANDEVA para la longitud (A) y biomasa orgánica (B) de larvas sometidas a diferentes salinidades y temperaturas. FV: fuente de variación, SC: suma de cuadrados, GL: grados de libertad, CM: cuadrados medios, F: estadístico, F: valor crítico de la distribución F, P: probabilidad / ANOVA for length (A) and organic biomass (B) of larvae under different salinities and temperature. FV: source of variation, $\mathrm{SC}$ : sum of squares, GL: degrees of freedom, CM: mean squares, $\mathrm{F}$ : statistical, $\mathrm{F}_{\mathrm{t}}$ : critical value of the $\mathrm{F}$ distribution, $\mathrm{P}$ : probability

\begin{tabular}{|c|c|c|c|c|c|c|c|}
\hline & FV & $\mathrm{SC}$ & GL & $\mathrm{CM}$ & $\mathrm{F}$ & $P$ & $\mathrm{~F}_{\mathrm{t}}$ \\
\hline \multirow[t]{5}{*}{ A } & Salinidad & 1,9303 & 3 & 0,6434 & 14,8998 & $1,8172 \times 10^{-6}$ & 2,8663 \\
\hline & Temperatura $\left({ }^{\circ} \mathrm{C}\right)$ & 9,2984 & 2 & 4,6492 & 107,6576 & $6,4492 \times 10^{-16}$ & 3,2594 \\
\hline & Interacción & 0,2478 & 6 & 0,0413 & 0,9562 & 0,4682 & 2,3637 \\
\hline & Residual & 1,5447 & 36 & 0,0432 & & & \\
\hline & Total & 13,0312 & 47 & & & & \\
\hline \multirow[t]{5}{*}{ B } & Salinidad & 712,9989 & 3 & 237,6663 & 44,0438 & $3,9020 \times 10^{-12}$ & 2,8663 \\
\hline & Temperatura $\left({ }^{\circ} \mathrm{C}\right)$ & 6749,3391 & 2 & 3374,6695 & 625,4089 & $1,1019 \times 10^{-28}$ & 3,2594 \\
\hline & Interacción & 651,2732 & 6 & 108,5455 & 20,1161 & $3,6983 \times 10^{-10}$ & 2,3637 \\
\hline & Residual & 194,2539 & 36 & 5,3959 & & & \\
\hline & Total & 8307,8651 & 47 & & & & \\
\hline
\end{tabular}


$\mathrm{Al}$ final del experimento en los tratamientos de $30-35^{\circ} \mathrm{C} \mathrm{y}$ salinidad entre 25-35 se obtuvieron organismos en la etapa de PL1 y cuyas longitudes promedio no fueron estadísticamente diferentes, encontrando en la combinación de $30^{\circ} \mathrm{C}$ y salinidad de 30 los individuos con la mayor longitud media de $4,15 \mathrm{~mm}$ y tasa diaria de crecimiento de $0,52 \mathrm{~mm} \mathrm{día}^{-1}$, seguida por los organismos del tratamiento de $30^{\circ} \mathrm{C}$ y salinidad 25 que tuvieron una talla promedio de $3,95 \mathrm{~mm}$ y tasa de $0,49 \mathrm{~mm} \mathrm{día}^{-1}$. Las tallas de estos 2 tratamientos mostraron diferencias significativas con las longitudes medias obtenidas en las combinaciones de $30-35^{\circ} \mathrm{C}$ y salinidad 40 (Tabla 2). Asimismo, las tallas promedio estimadas en los 4 tratamientos a $25^{\circ} \mathrm{C}$ fueron estadísticamente similares, pero diferentes a las longitudes obtenidas con las combinaciones de $30-35^{\circ} \mathrm{C}$. La menor longitud media y tasa diaria de crecimiento, 2,53 y 0,29 $\mathrm{mm}$ día $^{-1}$ respectivamente, se determinaron en el tratamiento de $25^{\circ} \mathrm{C}$ y salinidad 40 cuyas larvas tuvieron los menores desarrollos larvarios presentando estadios de zoea III y mysis I (Tabla 2).

Los individuos del tratamiento de $30^{\circ} \mathrm{C}$ y salinidad 35 en las fases de mysis III-PL1 tuvieron el mayor peso orgánico promedio final $(75,73 \mu \mathrm{g})$ y la tasa de crecimiento por día $(10,51$ $\left.\mu \mathrm{g} \mathrm{día}^{-1}\right)$; sin embargo, este valor de peso no fue estadísticamente diferente a los estimados con la combinación de $30^{\circ} \mathrm{C}$ y salinidad entre $25-30$, además de $35^{\circ} \mathrm{C}$ y salinidad 25 que superaron los $70 \mu \mathrm{g}$ por organismo y tasa diaria prácticamente de $10 \mu \mathrm{g} \mathrm{día}^{-1}$ (Tabla 2 ). Al igual que con las longitudes, los valores promedios menores de los pesos orgánicos finales se estimaron con los tratamientos a $25^{\circ} \mathrm{C}$ y mostraron diferencias significativas con las combinaciones restantes; aunque el valor más bajo en peso $(40,18 \mu \mathrm{g})$ y la menor tasa de crecimiento $\left(5,43 \mu \mathrm{g} \mathrm{día}^{-1}\right)$ se presentaron en los organismos cultivados a $25^{\circ} \mathrm{C}$ y salinidad 35 cuyas larvas alcanzaron el estadio de mysis I (Tabla 2).

Algunos investigadores también han señalado que la temperatura y la salinidad presentan un efecto significativo en la longitud durante las primeras etapas de vida de Penaeus semisulcatus De Haan, 1844 y en la biomasa de juveniles de Penaeus monodon Fabricius, 1798 y L. vannamei (Kumlu et al. 2000, Ye et al. 2009, Su et al. 2010). En el presente estudio, la mayor longitud y biomasa se encontraron a $30^{\circ} \mathrm{C}$ con salinidad entre 25-30. Otros peneidos mostraron las mejores longitudes y pesos entre $27-30^{\circ} \mathrm{C}$ y salinidad entre 27-35 (Kumlu et al. 2000, Ye et al. 2009, Pham et al. 2012).

Tabla 2. Valores finales del promedio y (desviación estándar) de longitud total, peso orgánico, tasa de crecimiento y etapa larval de Litopenaeus vannamei cultivado a diferentes combinaciones de salinidad y temperatura. Las letras diferentes en la columna de longitud y peso orgánico indican diferencias significativas $(\mathbf{P}<\mathbf{0 , 0 5}$ ) / Mean values and (standard deviation) of total length, organic weight, growth rate and larval development of Litopenaeus vannamei grown at different combinations of salinity and temperature. The different letters in the column of length and organic weight indicate significant differences $(P<0.05)$

\begin{tabular}{|c|c|c|c|c|c|c|}
\hline $\begin{array}{c}\text { Temperatura } \\
\left({ }^{\circ} \mathrm{C}\right)\end{array}$ & Salinidad & $\begin{array}{l}\text { Longitud total } \\
\qquad(\mathrm{mm})\end{array}$ & $\begin{array}{l}\text { Peso orgánico } \\
\left(\mu \mathrm{g} \text { larva }^{-1}\right)\end{array}$ & $\begin{array}{l}\text { Tasa de cr } \\
\left(\mathrm{mm} \mathrm{día}^{-1}\right)\end{array}$ & $\begin{array}{l}\text { ecimiento } \\
\left(\mu \mathrm{g} \mathrm{día}^{-1}\right)\end{array}$ & Etapa larval \\
\hline \multirow[t]{4}{*}{25} & 25 & $2,87(0,13)^{\mathrm{a}}$ & $41,68(1,84)^{\mathrm{a}}$ & 0,33 & 5,64 & MI \\
\hline & 30 & $3,00(0,04)^{\mathrm{ab}}$ & $45,19(2,96)^{\mathrm{a}}$ & 0,35 & 6,14 & MI \\
\hline & 35 & $2,85(0,13)^{\mathrm{a}}$ & $40,18(1,75)^{\mathrm{a}}$ & 0,33 & 5,43 & MI \\
\hline & 40 & $2,53(0,37)^{\mathrm{a}}$ & $40,27(2,79)^{\mathrm{a}}$ & 0,29 & 5,44 & ZIII-MI \\
\hline \multirow[t]{4}{*}{30} & 25 & $3,95(0,25)^{d}$ & $74,46(2,96)^{d}$ & 0,49 & 10,33 & PL1 \\
\hline & 30 & $4,15(0,19)^{\mathrm{d}}$ & $72,12(2,36)^{d}$ & 0,52 & 9,99 & PL1 \\
\hline & 35 & $3,69(0,14)^{\mathrm{cd}}$ & $75,73(3,75)^{d}$ & 0,45 & 10,51 & MIII-PL1 \\
\hline & 40 & $3,42(0,10)^{\mathrm{bc}}$ & $55,77(1,40)^{\mathrm{b}}$ & 0,41 & 7,66 & MIII \\
\hline \multirow[t]{4}{*}{35} & 25 & $3,86(0,19)^{\mathrm{cd}}$ & $70,05(2,29)^{\mathrm{d}}$ & 0,48 & 9,70 & PL1 \\
\hline & 30 & $3,77(0,28)^{\mathrm{cd}}$ & $61,42(1,74)^{\mathrm{bc}}$ & 0,46 & 8,46 & PL1 \\
\hline & 35 & $3,69(0,26)^{\mathrm{cd}}$ & $62,31(0,52)^{\mathrm{c}}$ & 0,45 & 8,59 & PL1 \\
\hline & 40 & $3,39(0,18)^{\mathrm{bc}}$ & $59,31(1,68)^{\mathrm{bc}}$ & 0,41 & 8,16 & MIII \\
\hline
\end{tabular}


Entre las combinaciones de salinidad-temperatura, los resultados indican que esta especie de camarón se adapta mejor a bajas salinidades, puesto que la longitud, el peso y desarrollo larvario fueron considerablemente menores a salinidad 40, en las temperaturas ensayadas. Aunque, se sabe que las larvas de L. vannamei pueden desarrollarse a salinidades entre 20 y 45 (Chong-Robles et al. 2014), debido a que es una especie eurihalina (Pan et al. 2007). Sin embargo, existen evidencias que las larvas de peneidos no toleran variaciones bruscas de salinidad y temperatura, debido a que sus branquias y otras estructuras especializadas en la osmorregulación no se han desarrollado completamente (Miranda et al. 2010, Pham et al, 2012, Arzola et al. 2013). Algunos estudios, indican que el desarrollo y crecimiento de P. merguiensis, P. semisulcatus y L. stylirostris durante las fases larvales es menor y más tardado a salinidad 25 en comparación de 30, 35 y 40, mantenidos a diferentes temperaturas de 29 y $34^{\circ} \mathrm{C}$ (Kumlu et al. 2000, Zacharia \& Kakati 2004, Pham et al. 2012). Asimismo, el incremento en peso en juveniles de $P$. monodon ha sido registrado menor a salinidad 5, 10, 15 en comparación de 20, 25 y 30 , mantenidos a una temperatura de $28,8^{\circ} \mathrm{C}$ (Ye et al. 2009). Otros informes revelan que en $30^{\circ} \mathrm{C}$ se incrementa de manera significativa la ganancia en peso de juveniles de $L$. vannamei en comparación de 20 y $25^{\circ} \mathrm{C}$, mantenidos a diferentes salinidades (Su et al. 2010). En este estudio las larvas presentaron los mejores parámetros (tanto en peso como en longitud) en las temperaturas de 30 y $35^{\circ} \mathrm{C}$ ( 7 días) lo cual corrobora la influencia que tiene este factor sobre el crecimiento, desarrollo y supervivencia de las larvas de camarones, así como de otros organismos marinos (Kumlu et al. 2000, Zacharia \& Kakati 2004, Azocar et al. 2014).

La incidencia de la temperatura y salinidad en el crecimiento de las primeras etapas de vida del camarón L. vannamei le confieren un rol importante entre las variables hidrológicas del agua, las cuales no pueden obviarse al momento de cultivar organismos acuáticos. De acuerdo a los resultados del presente estudio, se sugiere que las empresas comerciales que producen poslarvas de camarón, mantengan los cultivos en los intervalos de temperatura de $30-35^{\circ} \mathrm{C}$ y de salinidad de $25-35$.

\section{Agradecimientos}

A los proyectos UAS-CA-162, PROFAPI-159-2014 y al CONACYT por el financiamiento y beca asignada al primer autor (CVU 206165), a la proveedora de larvas FITMAR y Juan M. Flores-Alarcón.

\section{LITERATURA CITADA}

Arzola GJ, VP Piña, SM Nieves \& JM Medina. 2013. Supervivencia de postlarvas de camarón blanco Litopenaeus vannamei a diferentes salinidades y temperaturas. Revista de Medicina Veterinaria y Zootecnia-Córdoba 18: 3618-3625.

Azocar C, G Claramunt, F Yañez \& M Futagawa. 2014. Efecto de la temperatura sobre el desarrollo embrionario y larval de Graus nigra (Kyphosidae) del norte de Chile. Revista de Biología Marina y Oceanografía 49(1): 111-122.

Chong-Robles J, G Charmantier, V Boulo, J LizárragaValdéz, LM Enríquez-Paredes \& I Giffard-Mena. 2014. Osmoregulation pattern and salinity tolerance of the white shrimp Litopenaeus vannamei (Boone, 1931) during postembryonic development. Aquaculture 422/423: 261-267.

Godínez-Siordia DE, MC Chávez-Sánchez \& S GómezJiménez. 2011. Acuicultura epicontinental del camarón blanco del Pacífico, Litopenaeus vannamei (Boone, 1931). Tropical and Subtropical Agroecosystems 14: 55-62.

Hoang-Oanh DT, T Quoc-Phu, N Thanh-Phuong \& P AnhTuan. 2013. Estudios en Vietnam encuentran como responsables de la transmisión del EMS/AHPNS a un Vibrio y un fago. Industria Acuícola 9(5): 14-15.

Jamali H, N Ahmadifard \& D Abdollahi. 2015. Evaluation of growth, survival and body composition of larval white shrimp (Litopenaeus vannamei) fed the combination of three types of algae. International Aquatic Research 7: 115-122.

Kumlu M, OT Eroldogan \& M Aktas. 2000. Effects of temperature and salinity on larval growth, survival and development of Penaeus semisulcatus. Aquaculture 188: 167-173.

Laramore SE, J Scarpa, CR Laramore \& J Lin. 2009. Virulence variation of white spot syndrome virus in Pacific white shrimp Litopenaeus vannamei. Journal of Aquatic Animal Health 21: 82-90.

Magallón-Barajas FJ, R Servín-Villegas, G Portillo-Clark \& B López-Moreno. 2006. Litopenaeus vannamei (Boone) post-larval survival related to age, temperature, $\mathrm{pH}$ and ammonium concentration. Aquaculture Research 37: 492-499.

Medina-Jasso MA, JF Arzola-González, P Piña-Valdez \& M Nieves-Soto. 2015. Efecto de la dieta tradicional y no tradicional en la respiración y excreción de larvas de camarón blanco Litopenaeus vannamei. Revista de Medicina Veterinaria y Zootecnia-Córdoba 20: 4917-4928.

Miranda I, JL Valles, R Sánchez \& Z Álvarez. 2010. Cultivo del camarón marino Litopenaeus vannamei (Boone, 1931) en agua dulce. Revista Científica de la Facultad de Ciencias Veterinarias de la Universidad del Zulia 20(4): 339-346. 
Pan L, L Zhang \& H Liu. 2007. Effects of salinity and pH on ion-transport enzyme activities, survival and growth of Litopenaeus vannamei postlarvae. Aquaculture 273: 711720.

Pham D, G Charmantier, N Wabete, V Boulo, F Broutoi, J Mailliez, J Peignon \& M Charmantier-Daures. 2012. Salinity tolerance, ontogeny of osmoregulation and zootechnical improvement in the larval rearing of the Caledonian Blue Shrimp, Litopenaeus stylirostris (Decapoda, Penaeidae). Aquaculture 362-363: 10-17.

Piña P, D Voltolina, M Nieves \& M Robles. 2006. Survival, development and growth of the Pacific white shrimp Litopenaeus vannamei protozoea larvae, fed with monoalgal and mixed diets. Aquaculture 253: 523-530.

Piña-Valdez P, JF Arzola-González, M Nieves-Soto \& MA Medina-Jasso. 2015. Efecto combinado de temperatura y salinidad en el consumo de oxígeno en postlarvas de camarón blanco Litopenaeus vannamei. Boletim do Instituto de Pesca 41(1): 89-101.
Rodríguez-Canché LG, TD Maldonado-Montiel \& LA Carrillo-Navarro. 2006. Calidad biológica y bioquímica de la población de Artemia (Anostraca: Artemiidae) localizada en las salinas de Real de Salinas, Calkini, Campeche, México. Revista de Biología Tropical 54(4): 1283-1293.

Su Y, S Ma \& C Feng. 2010. Effects of salinity fluctuation on the growth and energy budget of juvenile Litopenaeus vanname $i$ at different temperatures. Journal of Crustacean Biology 30(3): 430-434.

Ye L, S Jiang, X Zhu, Q Yang, W Wen \& K Wu. 2009. Effects of salinity on growth and energy budget of juvenile Penaeus monodon. Aquaculture 290: 140-144.

Zacharia S \& VS Kakati. 2004. Optimal salinity and temperature for early developmental stages of Penaeus merguiensis De man. Aquaculture 232: 373-382.

Zar JH. 2010. Biostatistical analyis, 944 pp. Prentice-Hall, Upper Saddle River.

Recibida el 3 de octubre de 2016 y aceptada el 21 de agosto de 2017

Editor: Claudia Bustos D. 